\title{
Reformulation, négociation et progression discursive dans le débat radiophonique. Enjeux pour le développement de l'enseignement/apprentissage de la compétence discursive
}

\author{
Reformulation, negotiation and progression \\ in French radio programme. \\ Teaching and learning oral speech skills in the classroom
}

\author{
Élisabeth Richard \\ Université Rennes 2 LIDILE EA 3874 \\ elisabeth.richard@univ-rennes2.fr \\ Griselda Drouet \\ Université Rennes 2 LIDILE EA 3874 \\ Griselda.drouet@uhb.fr
}

\begin{abstract}
In this article, we deal with listening comprehension skills as we would like to define it anew in light of an oral speech analysis perspective insisting on developing the communicative expertise. In order to do so, we rely on a methodology that implies observation and analysis of oral speech and that highlights the language dynamics and the multiple enunciative point of views structuring radio programme debates.
\end{abstract}

Keywords: glottodidactics, listening comprehension skills, oral speech, FLE, debate, radio programmes, interaction.

\section{INTRODUCTION}

Si l'un des objectifs de la glottodidactique est « d'appréhender une vision claire de l'objet à enseigner (...) pour mettre en place des modalités d'enseignementapprentissage » opérationnelles (Górecka, Wojciechowska, ici-même), il faut alors 
explorer le discours en contexte afin 1) d'en mettre au jour les spécificités, 2) d'en souligner les dynamiques syntaxiques et énonciatives pour 3) en proposer une didactique étayée. L'objet de notre recherche se situe aux confins de l'analyse syntaxique et énonciative de la langue à l'oral et de la didactique de la compréhension orale. Il s'agit pour nous de mettre au jour une didactique qui s'appuie sur une meilleure appréhension des dynamiques à l'œuvre dans le flux de la langue orale et qui prend en compte les diverses postures énonciatives qui sous-tendent les enjeux d'une interaction.

Dans le cadre de cet article, nous proposons de redéfinir la compétence de compréhension orale en l'inscrivant dans une perspective discursive du développement des compétences de communication langagières. Nous nous basons, pour ce faire, sur une méthodologie d'observation et d'analyse de la langue orale qui prend en compte les dynamiques discursives et les postures énonciatives qui se jouent dans un débat radiophonique.

\section{CONSTATS PRÉLIMINAIRES}

\subsection{DÉPASSER LES IDÉES REÇUES SUR LA LANGUE ORALE}

Les idées reçues sur la langue orale sont tenaces et les avancées des recherches ont bien du mal à entrer, ou à trouver écho en didactique du français langue étrangère (désormais FLE). Les spécificités de la langue orale communément admises restent les mêmes dans les manuels et autres outils pédagogiques : réduction des syllabes, chute du E caduc, absence de double marquage de la négation, lexique et, surtout, niveau de langue spécifiques. Pourtant, étudier ou montrer la langue orale ne peut pas se restreindre à l'étude des variations de registre, variations trop vite schématisantes et rapportant l'oral à des faits de non-conformité.

Mais alors comment «montrer» la langue orale ? Et comment montrer ses spécificités, non pas seulement d'ordre phonétique et prosodique (ce qui reste très important, même pour les niveaux très avancés), mais aussi, et peut-être surtout, ses spécificités d'ordre communicatif et discursif? Cette question traverse depuis quelques années notre réflexion.

Le point de départ de cette réflexion est double : linguistique et didactique. En travaillant avec les didacticiennes de l'université Adam Mickiewicz de Poznań (Pologne), nous avons pris conscience que chez des apprenants de niveau avancé (C1/C2), il reste difficile de dépasser la démarche de restitution omniprésente dans les tâches d'écoute jusqu'aux niveaux B1/B2. Nous avons observé que ces tâches d'écoute, qui privilégient le repérage et l'accumulation des informations, ne prennent pas suffisamment en compte le caractère non linéaire, dynamique et coconstruit du discours oral (Richard et al., 2012). Par ailleurs, l'analyse des consignes 
d'activités d'écoute rend compte de l'insuffisance des propositions didactiques concernant l'oral radiophonique, propositions qui restent le plus souvent calquées sur les textes écrits (de type «articles de presse»Wojciechowska et al., 2014). Ces activités imposent une démarche de compréhension basée sur les aspects linguistiques, textuels et référentiels, certes, mais sans les intégrer dans une construction plus large et sans tenir suffisamment compte de la dimension polémique, sociale et finalisée (intentionnelle) du discours (Wojciechowska, 2017). Du côté de la formation des professeurs de FLE, nous constatons également que les (futurs) enseignants que nous formons n'ont de représentation de la langue orale que celle que leur a toujours dictée l'école : une langue imparfaite, incomplète, répétitive, médiocre, voire vulgaire ; une langue à éviter, et qu'ils n'ont jamais vraiment écoutée.

\subsection{MÉTHODOLOGIE}

Notre choix méthodologique de départ s'est porté sur l'étude des débats radiophoniques longs, à plusieurs voix, pour tenter de répondre aux exigences du Cadre Commun de référence pour les langues (désormais CECRL), qui rappelle qu'un apprenant autonome de niveau $\mathrm{C} 1$ «comprend des documents oraux longs et exigeants » et qu'un locuteur expert de niveau $\mathrm{C} 2$ «comprend tout et sans effort » (p. 25) ${ }^{1}$.

Force est de constater que même chez les étudiants francophones natifs, l'écoute attentive et suivie d'une émission type Le téléphone sonne demande un effort conséquent qu'ils ne sont pas tous capables de tenir. Nous pouvons aujourd'hui affirmer que ce qui fait la difficulté majeure, c'est 1) le faible taux d'écoute de débats radiophoniques chez nos étudiants, en Pologne comme en France et 2) leur méconnaissance du déploiement de la langue à l'oral. En outre, les étudiants comme les apprenants s'arrêtent dans leur écoute sur les éléments ponctuels qu'ils ne mâ̂trisent pas, en particulier les noms propres, et échappent complètement à la progression du discours qui se joue dans le débat à la fois chez un seul locuteur et a fortiori entre les interlocuteurs. Ils cherchent à restituer les contenus tels qu'ils ont été produits par un locuteur sans prendre en compte le caractère ou le contexte social et institutionnel de l'énoncé, son histoire interdiscursive - saisissable notamment à travers le jeu des contenus implicites (Richard, Wojciechowska, 2016) - et sa dimension pragmatique.

Si nous voulons habituer les apprenants à comprendre le genre débat radiophonique, il nous semble qu'il faut d'abord les habituer à comprendre les mécanismes propres à la forme dynamique de la langue à l'oral. Une fois initiés, les apprenants

\footnotetext{
${ }^{1}$ http://www.coe.int/t/dg4/linguistic/Source/Framework_fr.pdf
} 
seront en capacité de repérer les indices du débat à l'oral et d'isoler les rôles énonciatifs différents qui émergent à des moments de discours distincts à l'intérieur même d'une émission radiophonique à plusieurs intervenants. Si nous voulons dépasser les instructions du niveau B2, nous devons mener les apprenants au-delà du contenu et de la restitution du continu, pour découvrir toutes les dynamiques à l'œuvre dans le discours en interaction. En somme, ce que les apprenants doivent apprendre à observer, ce n'est pas tant le contenu que la progression, les accroches, les failles, les implicites et les aspérités du discours. L'objectif in fine est de déchiffrer la subtilité énonciative de la polémique.

Les questions que les apprenants doivent alors apprendre à se poser sont les suivantes : Où en est-on dans le débat? Qu'est-ce qui est nouveau dans ce débat ? Comment en est-on arrivé là ? Qu'est-ce qui mérite d'être dit? Qu'est-ce qui fait polémique?

Dans cette optique, nous nous sommes donc fixées deux objectifs parallèles :

1) montrer les mécanismes propres à la progression du discours à l'oral et chercher des traces d'une collaboration discursive qui démontrent la coconstruction du discours à l'oral dans un débat radiophonique,

2) rendre explicites des routines langagières liées aux genres de discours à l'oral et aux rôles énonciatifs des locuteurs.

Dans le présent article, nous avons fait des choix qui, sans y être totalement opposés, ne remplissent pas exactement les conditions méthodologiques fixées, mais qui permettent néanmoins d'accéder à ces premiers objectifs. En effet, nous avons préféré travailler sur une émission assez courte (19 minutes au lieu des 55/60 que dure une émissions type Le téléphone sonne $e^{2}$; nous avons aussi pris le parti d'observer la dynamique à l'œuvre dans un discours oral entre deux locuteurs seulement et non, comme nous nous l'étions imposé au départ entre plusieurs $(4,5)$ intervenants; enfin, nous nous sommes concentrées sur une émission grand public, dans laquelle un journaliste reçoit un seul invité qui vient présenter son actualité, ici un géographe qui vient de publier un ouvrage de géographe. Il s'agit de l'émission L'invité des matins du 13 septembre 2016, animée par le journaliste Guillaume Erner sur France Culture, qui s'intitule «La faillite des élites » et dont l'invité en est Christophe Guilluy.

A priori donc, nous nous éloignons du débat, en tout cas, du débat tel qu'il avait été défini au début de notre projet. Mais ce choix d'une émission à deux intervenants et sans polémique apparente, vise une meilleure appréhension de la langue à l'oral et de la progression négociée (Kerbrat-Orecchioni, 2012) qui s'y joue.

\footnotetext{
${ }^{2}$ Émission française de radio diffusée quotidiennement sur France Inter qui aborde chaque jour un thème de l'actualité en invitant des personnalités. Les auditeurs peuvent alors entrer en interaction avec le présentateur et ses invités grâce au standard téléphonique, à la messagerie électronique de l'émission ou aux réseaux sociaux.
} 


\section{CIRCULARITÉ ET DYNAMIQUES DE L'ORAL}

\subsection{CHEZ UN LOCUTEUR, LE DISCOURS AVANCÉ}

Écouter la langue orale ne va pas de soi. Non pas parce qu'elle serait désarticulée, désorganisée, mais parce que son organisation révèle une planification très différente de ce que nous a imposé l'écrit avec ses modèles de phrases, de paragraphes et d'enchaînements assez contraints, d'ailleurs nommés traditionnellement «enchaînements logiques ». Suivre un discours oral demande d'être attentifs non seulement $\mathrm{au}(\mathrm{x})$ contenu(s), mais aux ruptures qui s'y jouent, comme le souligne l'extrait suivant (1) :

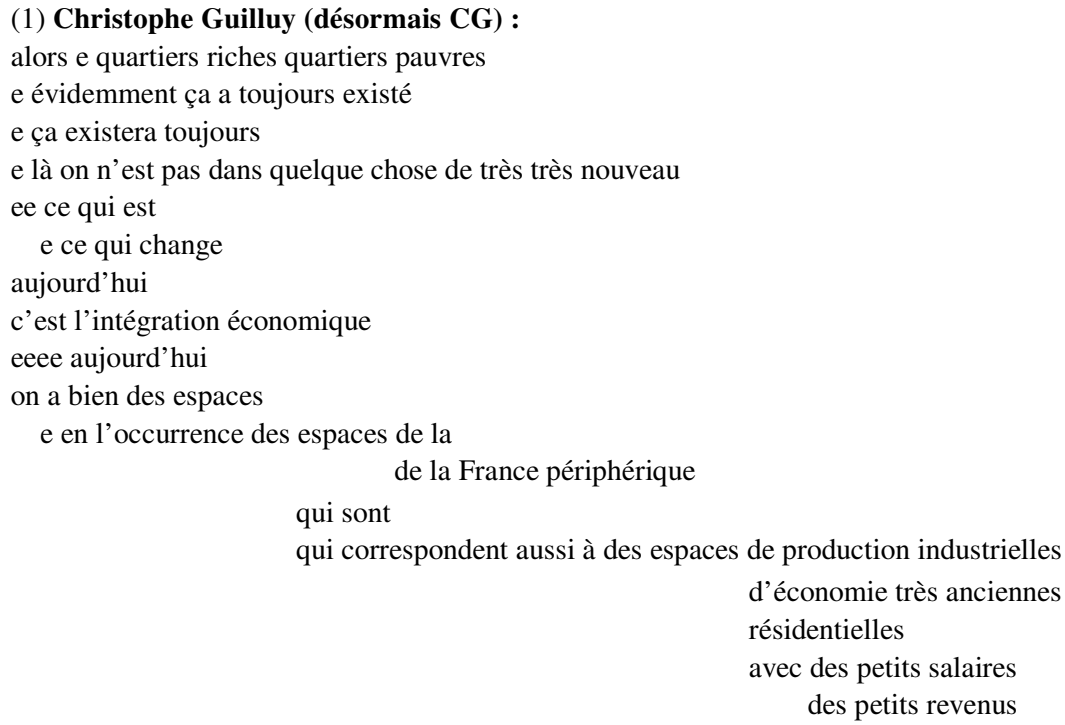

et inversement

une très forte concentration de l'emploi sur sur les catég sur sur les grandes dans les grandes métropoles 
Nous avons là, la transcription de 53 secondes de paroles. Le flux est continu mais la progression n'est pas linéaire, elle avance comme par tâtonnement et reformulations diverses. Il faut apprendre à repérer et à se repérer dans le dédale a priori de ces nombreuses ruptures et enchâssements multiples. La langue orale est une langue de l'élargissement. Les travaux de Claire Blanche Benveniste (1990) l'ont très bien montré. Et la mise en grille syntaxique qu'elle propose, que nous utilisons ici, est à notre avis un très bon modèle didactique de visualisation des dynamiques internes spécifiques.

Il s'agit d'habituer l'apprenant à isoler ce qui fait rupture et continuation, à différencier les reformulations des accumulations et à repérer les indices qui signalent la sortie des incises et des parenthèses, c'est-à-dire à repérer ce qui reprend le fil du discours et délimite ainsi les frontières entre discontinuités et propos/information principal/e comme nous le voyons en (2) :

(2) donc c'est ces catégories populaires

e et effectivement je me suis posé la question

bé e finalement

ces catégories populaires aujourd'hui c'est quoi

ce sont des ouvriers

ce sont employés

ce sont des gens qui vivent en dessous du du du revenu médian hein

le le le niveau de vie médian en France

c'est mille six cents

on est vraiment sur des catégories vraiment très très modestes

mille sept cents euros enfin ??

et puis ça peut aller jusque effectivement des ménages pauvres

et l'ensemble de ces catégories

des ménages pauvres

elles sont majoritaires

très majoritaires hein

e si je je prends l'ensemble des catégories e modestes et populaires c'est $60 \%$ de la population active

e 55 en réalité si je

je

je

j'isole simplement les ouvriers et les employés

e hier y'avait plus d'ouvriers

y'a moins d'ouvriers

mais bon

y'a plus d'employés aujourd'hui

on est bien sur des catégories très très modestes

ce qui est intéressant c'est de voir que

e ben elles ne vivent plus e exactement au même endroit e aujourd'hui qu'hier

et qu'est-ce qui s'est passé

ben $\mathrm{j}$ ' allais dire que

très e très cyniquement finalement

on s'aperçoit que finalement le

ben finalement le le marché immobilier

e les logiques économiques se se sont chargés de

finalement d'évacuer les gens

e qui n'étaient pas utiles pour l'économie 
Ces mises en grille permettent également de mieux se rendre compte de la circularité du discours à l'oral. En effet, outre les enchâssements de complémentation multiples (relatives, complément du nom, juxtaposition), nous repérons qu'un des schémas syntaxiques les plus récurrents à l'oral (en tout cas dans ce type de débat radiophonique) est un schéma en deux temps, sur le modèle des clivées/pseudoclivées, d'une part comme en (1') ou (2'a), ou les dislocations, comme en (2'b) :

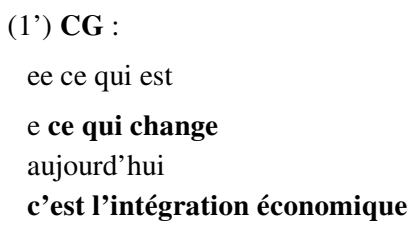

(2'a) CG :

mais bon

on est bien sur des catégories très très modestes

ce qui est intéressant c'est de voir que

e ben elles ne vivent plus e exactement au même endroit e aujourd'hui qu'hier

(2’b) CG :

et l'ensemble de ces catégories

elles sont majoritaires

Les grammaires et autres manuels ont depuis longtemps repéré les clivées et les dislocations comme des schémas de l'oral, mais le plus souvent c'est pour les rapporter au seul principe d'expressivité de la langue orale. Or, nous nous apercevons, en analysant la langue orale de ces locuteurs experts de la parole publique, que ces choix syntaxiques révèlent avant tout une stratégie énonciative particulière : le locuteur dit ce qu'il a à dire, immédiatement, et une grande partie de son temps de parole est utilisée à expliquer, justifier, exemplifier ce qu'il a de toute façon déjà posé comme singulier. Nous sommes ici dans une rhétorique argumentative par démonstration : une information se voit posée puis décomposée et recomposée. Tout le jeu du locuteur-expert, c'est de savoir rebondir et revenir là où il en était.

Les répétitions segmentales (surlignées dans les transcriptions de l'exemple (2)) sont ainsi à analyser comme des indices non de stagnation mais de progression du discours, on pourrait dire comme des indices de paliers dans la chaîne énonciative et informative : à chaque fois qu'un segment est répété, l'auditeur doit interpréter qu'une nouvelle information vient d'être donnée et qu'une autre (toute aussi nouvelle et spécifique) va l'être. 


\subsection{ENTRE LES LOCUTEURS : L'HISTOIRE DISCURSIVE SE CONSTRUIT}

Du côté de l'interaction, le discours progresse aussi. Nous trouvons, ponctuellement mais régulièrement, des indices qui permettent à l'auditeur de comprendre qu'on fait toujours partie du même discours et qu'on a construit, ensemble, une histoire discursive particulière.

De façon assez simple, nous pensons aux interventions du journaliste qui rappelle le nom de l'invité et le titre de son ouvrage, raccrochant et délimitant ainsi systématiquement les différentes interventions à ce cadre discursif spécifique. Sur l'ensemble des tours de parole que comptent les 19 minutes d'entretien, le journaliste rappelle l'objet du jour 9 fois :

(3) Journaliste (désormais JOUR) :

JOUR : Bonjour Christophe Guilluy

CG : Bonjour

JOUR : Vous publiez la France d'en haut

c'est un livre qui vient donc six ans après fracture française

qu'est-ce qui s'est passé dans ces six ans

JOUR : alors pour ceux qui ne vous connaissent pas

hein Christophe Guilluy

vous êtes géographe

JOUR : mais alors on se pose la question suivante en vous lisant

JOUR :

c'est-à-dire que

pour essayer de vulgariser Le Guilluy

e autrement dit

\section{JOUR :}

et vous publiez donc

dans ce livre

le crépuscule de la France d'en haut

un certain nombre de cartes

JOUR :

alors moi qui vous ai lu

par exemple

je me dis

JOUR : (...)

l'enjeu central de votre livre consiste à dire que 
JOUR :

et là

je cite un extrait de votre dernier ouvrage

Christophe Guilluy

du Le crépuscule de la France d'en haut

\section{JOUR :}

on va continuer à en parler avec vous

Christophe Guilluy

à 8 h 15

vous publiez la crépuscule le crépuscule

pardon de la France d'en haut /

Les adverbes effectivement ou bien comme en (2"'a) et (2'b), jouent aussi un rôle dans la construction de l'histoire discursive : ils sont à interpréter comme des « indices d'une construction discursive collaborative, intégrative dans le sens où ils instaurent la prise en compte des propos tenus au sein même du débat radiophonique » (Wojciechowska, Richard, 2016, par. 27).

(2"'a) donc c'est ces catégories populaires

e et effectivement je me suis posé la question

bé e finalement

ces catégories populaires aujourd'hui c'est quoi

$(2$ '’b) on est bien sur des catégories très très modestes

Enfin, d'autres patrons sont utilisés, parfois tout à fait ponctuellement mais dont l'enjeu majeur est de dire qu' on fait partie d'une histoire discursive en construction : dans notre extrait, le géographe utilise des segments du type : «on est là encore », « je rebondis sur la carte » comme en (4) et (5):

(4) CG :

ces catégories modestes vivent très loin des euh des zones qui comptent aujourd'hui

euh y compris pour les médias

et ils disparaissent euh des écrans radar

mais là encore

on est sur une conséquence économique

qui est la sortie de la classe moyenne de toutes ces catégories modestes 


\section{(5) JOUR :}

et vous publiez donc dans ce livre le crépuscule de la France d'en haut un certain nombre de cartes alors y'a notamment une carte où on voit e la France qui barrée par une verticale une verticale qui part des Pyrénées c'est une verticale verte parce qu'elle représente finalement les zones où le mètre carré est le moins cher où il est équivalent à moins de mille euros le mètre carré y'a par exemple e la Haute Marne on écoute un documentaire un extrait d'un documentaire la France d'en face diffusé en octobre 2013 justement consacré à la Haute Marne

[... Intermède documentaire]

\section{JOUR :}

votre réaction Christophe Guilluy

CG :

alors là

on est e

on est au cœur du sujet

c'est-à-dire que

vous avez e aujourd'hui

e des catégories modestes

qui vivent e dans des zones d'emploi les moins actives

alors

quand on explique ça

les libéraux vous expliquent que

bé ben bon c'est pas grave

e vous n'avez qu'à faire comme aux États-Unis

e déménager

e être mobile

bon ee

sauf que

et je rebondis sur le la carte effectivement des prix au mètre carré

Ces segments signalent un espace-temps du discours, comme une étape, et raccrochent ainsi deux pans du scénario discursif qui pourraient sembler ou trop éloignés dans la chaîne énonciative ou trop différents, ou les deux. Ces formes de transitions-rappels mériteraient d'être étudiées plus avant non seulement parce qu'elles sont spécifiques à la langue orale, mais encore parce qu'elles donnent aussi au locuteur une posture énonciative de force, de locuteur expert, capable de naviguer, voire de contrôler les méandres du débat jusqu'à s'en démarquer en rejetant la polémique à un autre espace de discours, comme en (6) : 
(6) CG :

alors ça

ça

c'est encore un autre

c'est un autre sujet

la question du rapport à l'autre dans la société multiculturelle

on est encore sur un autre

e un autre

un autre débat

\section{TOURS DE PAROLES ET POSTURES ÉNONCIATIVES}

Dans l'émission radiophonique choisie, les rôles des locuteurs sont immédiatement repérés et repérables. Ils sont posés dès l'introduction du journaliste qui fait l'interview de son invité, géographe et essayiste. Pourtant, ces deux rôles discursifs distincts de journaliste et d'invité-spécialiste s'enrichissent et se modifient au cours de l'émission.

Les amorces des tours de paroles sont un lieu d'écoute privilégié pour dégager des rôles énonciatifs distincts, à la fois pour le journaliste et pour l'invité-géographe. Dans la circulation de la langue orale, en effet, les postures énonciatives se coconstruisent, se répondent en miroir et nous rencontrons les mêmes postures chez les deux locuteurs.

Trois postures énonciatives principales sont ici mises au jour: la posture de l'énonciateur descripteur, la posture de la justification et celle de l'énonciateurdébatteur.

\subsection{LA POSTURE DE L'ÉNONCIATEUR-DESCRIPTEUR}

La posture de l'énonciateur-descripteur est la posture-type du journaliste présentateur qui doit décrire et présenter son invité et son ouvrage. C'est d'ailleurs ainsi que journaliste commence l'émission :

(7) JOUR : Bonjour Christophe Guilluy

CG : Bonjour

JOUR : Vous publiez la France d'en haut

c'est un livre qui vient donc six ans après fracture française

qu'est-ce qui s'est passé dans ces six ans 
Il nomme successivement son invité et le titre de son ouvrage d'actualité. Puis le journaliste décrit le statut de géographe de son invité en (8) :

(8) JOUR : alors pour ceux qui ne vous connaissent pas hein Christophe Guilluy vous êtes géographe

et e vous utilisez une méthode qui e puise e auprès de la cartographie auprès de la démographie pour tenter donc d'aboutir à un diagnostic de l'état du territoire

Le géographe-invité lui aussi endosse le rôle de descripteur en rebondissant sur le tour de paroles du journaliste, il décrit son travail et ses ouvrages en (9) :

(9) CG :

je dirais que

dans ces six ans

e toutes les dynamiques

e que j'avais identifiées

e bon dans fracture française mais aussi dans la

la phase des nouvelles fractures sociales

que

que j'avais réalisé avec e

avec e Christophe Noyer

se sont renforcées

se sont cristallisées

on est bien sur des dynamiques de recomposition des territoires

qui vont

ben effectivement des grandes métropoles jusqu'aux zones rurales hein

parce que l'intérêt de la nouvelle géographie sociale

telle que je la décris

c'est effectivement

de décrire toutes les dynamiques et toutes les interactions entre les territoires

de ne pas isoler e les grandes villes d'un côté ou les banlieues d'un côté

les zones rurales de l'autre

Mais très vite, le journaliste et le géographe changent de posture. Il s'agit pour eux alors d'asseoir chacun leur légitimité à intervenir dans cette émission. C'est la posture de la justification. 


\subsection{LA POSTURE DE LA JUSTIFICATION}

Le journaliste assoit sa légitimité d'intervieweur en se posant en lecteur de l'ouvrage de son invité, il a des choses à en dire et il a des éléments à interroger comme de (10) (11) et (12) :

(10) JOUR : mais alors on se pose la question suivante en vous lisant

(11) JOUR :

c'est-à-dire que

pour essayer de vulgariser Le Guilluy

e autrement dit

(12) JOUR :

alors moi qui vous ai lu

par exemple

Le géographe justifie également sa présence à l'antenne, son rôle dans l'échange comme en (13) :

(13) CG :

oui alors e bon

je travaille évidemment sur l'ensemble des des des recensements de l'INSE

e évidemment sur des enquêtes de terrain

puisque je travaille beaucoup avec des collectivités sur des diagnostics

ee bref l'idée

c'est pour moi

non pas de travailler sur le territoire

déjà que le territoire ça veut pas dire grand-chose

e je suis géographe mais en réalité je crois pas au territoire

je crois aux gens

c'est-à-dire que e moi mon sujet depuis le début

c'est de travailler sur les catégories modestes

Dans un premier temps, il définit son statut comme conforme à ce qui est a prio$r i$ attendu d'un géographe grâce notamment aux marqueurs discursifs évidemment et puisque. Ensuite, il met en place une stratégie énonciative visant à imposer sa singularité (c'est pour moi), d'abord par la négative (non pas de travailler sur le territoire / ça veut pas dire grand chose) pour finalement asseoir son originalité par rapport à d'autres (je suis, je crois, moi mon sujet) et sa thèse propre (travailler sur les catégories modestes). La progression énonciative qui se joue ici amorce l'argumentation du débat à venir. Une fois posée, de part et d'autre, la légitimité de la prise de parole, le débat peut enfin commencer. 


\subsection{LA POSTURE DE L'ÉNONCIATEUR-DÉBATTEUR}

\subsubsection{LE JOURNALISTE}

Le journaliste dans la posture du débatteur est incisif : il initie et entretient la vraie/fausse polémique selon diverses stratégies. Il essaie de mettre son invité en défaut, de manière plus ou moins directe.

Il le fait soit de façon directe, frontale, comme en (14) :

(14) JOUR :

attendez

parce que je

je

je crois que vous allez un peu vite en besogne

en fait e

soit de manière partiellement détournée, avec ici le marqueur de contradiction indirecte quand même (Drouet, sous presse ; Alvarez-Prendez, 2004), en (15) :

(15) JOUR : mais alors on se pose la question suivante en vous lisant

on se dit

finalement

ça n'est quand même pas très nouveau qu'il y ait des quartiers riches et des quartiers pauvres

soit il le fait de manière indirecte, implicite, et ces cas seront évidemment les plus compliqués à décrypter pour les apprenants. Sous couvert de résumer ou de synthétiser l'ouvrage de son invité, le journaliste-débatteur remet en question certains arguments du géographe.

Deux cas de figures se présentent alors : soit l'implicite se construit avec des marqueurs comme en (16), soit il se construit sans aucun marqueur particulier, comme en (17) :

\section{(16) JOUR :}

finalement

l'immigration est un choix intéressant

pour une grande majorité de français

sauf pour ces catégories modestes

qui

selon vous

sont reléguées

et en quelque sorte remplacées

si je vous suis bien

par ces immigrés \# 
CG :

oui euh

enfin bon

je vois où voulez en venir

\section{(17) JOUR :}

alors moi qui vous ai lu

par exemple

je me dis qu'il y a des zones autour des grandes métropoles

où le prix du mètre carré est très élevé

qui sont des zones où le prix du mètre carré est très abordable

par exemple le 93 pour la région parisienne

En (16), les indices de l'implicite sont nombreux, la présence de plusieurs marques de précautions énonciatives le souligne : selon vous / en quelque sorte / si je vous suis bien. L'auditeur est préparé à l'implicite, il est donc plus à même de l'interpréter.

En revanche en (17), la feinte du journaliste est plus subtile. Elle sera de fait plus difficile à repérer et à interpréter pour l'apprenant car elle n'est pas marquée dans le discours. L'énonciateur-débatteur se pose ici en faux naïf (Berrendonner, 2002) en énonçant un argument fallacieux. L'implicite ressortit ici du culturel et aucun indice linguistique dans la langue ne permet apriori de le repérer.

\subsubsection{LE GÉOGRAPHE}

À son tour, le géographe use des mêmes stratégies énonciatives.

Il débat en soulevant de manière directe l'implicite du journaliste comme en (18) :

(18) JOUR :

Inadapté

et dans cette troisième zone

alors là e

on trouve e une population

qui est une population reléguée

et oubliée en quelque sorte

à la fois des politiques publiques mais aussi du discours médiatique

CG :

oui alors

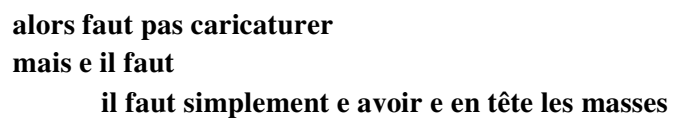

il faut simplement e avoir e en tête les masses 
Ce faisant, l'implicite est dénoncé. L'invité le met au jour et de le fait tomber, ce qui lui permet de reprendre la main pour imposer son argument : il faut simplement avoir en tête les masses.

Nous obtenons le même effet polémique, en (19), avec en plus mise en suspens de l'argumentation :

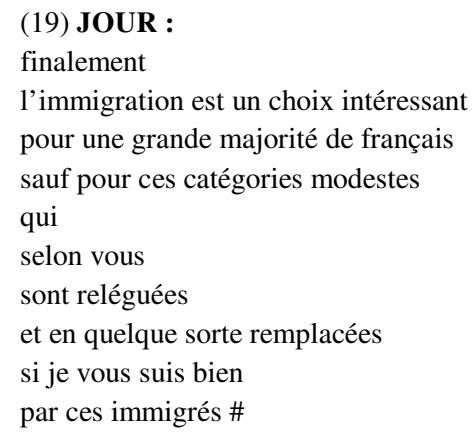

CG :

oui euh

enfin bon

je vois où voulez en venir

c'est

c'est

ça s'est pas passé comme ça /

L'invité peut également ne considérer qu'une partie de la contre-argumentation du journaliste en opérant une esquive partielle de l'implicite, comme en (20) :

\section{(20) JOUR :}

alors moi qui vous ai lu

par exemple

je me dis qu'il y a des zones autour des grandes métropoles

où le prix du mètre carré est très élevé

qui sont des zones où le prix du mètre carré est très abordable

par exemple le 93 pour la région parisienne

\section{CG :}

alors après

y'a les quartiers et communes de logements sociaux

on est là sur des concentrations hein

e mais en aucun cas eee le marché privé

permet aujourd'hui e l'arrivée de catégories modestes dans les grandes métropoles

c'est terminé 
Le marqueur après est ici à la fois concessif «c'est vrai » et polémique «mais ». Nous retrouvons alors le même phénomène en (21) :

\author{
(21) JOUR : \\ mais c'est \\ d'un autre côté \\ la crise de \# \\ CG : \\ bien sûr non mais \\ ce que je veux dire \\ c'est que \\ il faut partir euh de du structurel
}

avec la locution bien sûr associée à non mais, accord et désaccord partiels sont dits, qui, dans le même temps, garantissent et assurent la continuité du discours et son rebondissement.

Le géographe est également débatteur de sa propre pensée, comme en (22) :

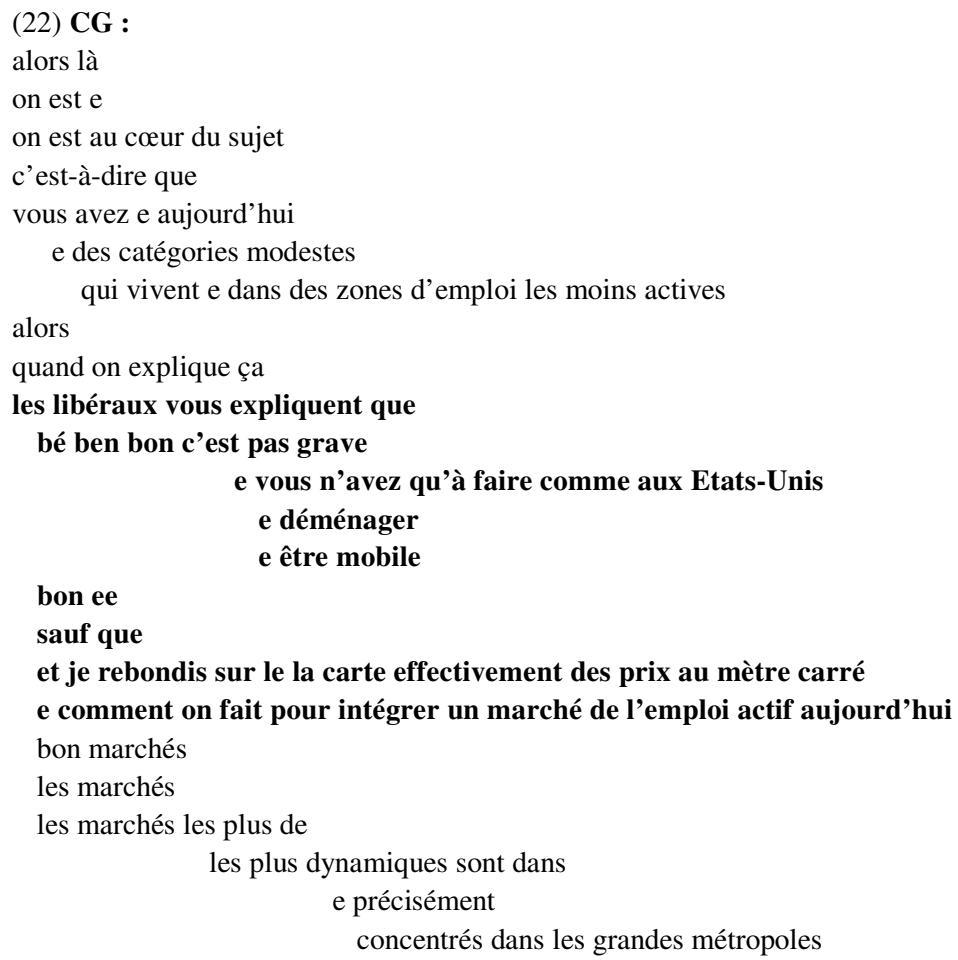


ou encore comme en (23):

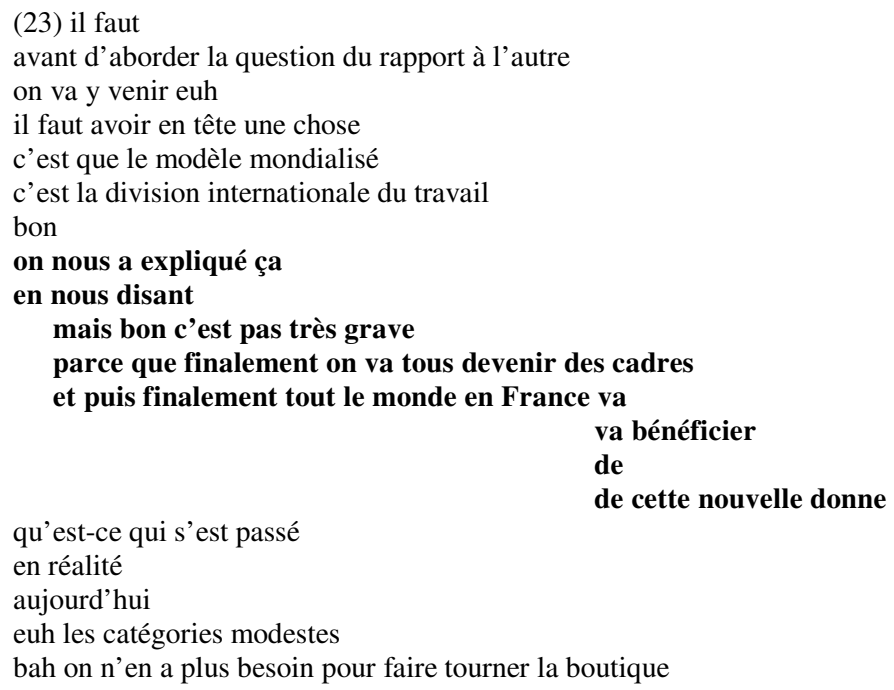

En mettant en scène du discours polyphonique à l'intérieur de sa propre intervention, il s'oppose à la voix de ses adversaires autres, politiques notamment, ce qui lui permet d'asseoir sa voix et sa thèse propres.

\section{RETOUR AUX POSTURES DE DESCRIPTEURS}

À la fin de l'émission, le journaliste et l'invité reprennent leur posture énonciative initiale de descripteurs :

\section{(24) JOUR :}

on va continuer à en parler avec vous

Christophe Guilluy

à $8 \mathrm{~h} 15$

vous publiez la crépuscule

le crépuscule

pardon

de la France d'en haut /

un livre avec également des propos assez durs contre les XXX

on va revenir là-dessus

sur une forme d'idéologie dominante de bien-pensante

$$
\text { de bien-pensance }
$$

vous expliquerez pourquoi vous en êtes arrivés à écrire ces propos assez durs \#

CG : pourquoi j’en suis arrivé là 
La boucle de l'interaction se referme, le scénario discursif est circulaire. C'est bien le journaliste qui ouvre et ferme le débat. Il a un rôle supplémentaire que l'invité n'a pas : celui de maitre de cérémonie. Au final, c'est bien lui qui décide, dans les grandes lignes, le tour que va prendre le scénario discursif. S'il n'a pas toujours la main sur l'information donnée au cours de l'interaction, il a bien en revanche la maîtrise du cadre discursif.

\section{CONCLUSION}

Entre le journaliste et le spécialiste, la parole passe, se passe, se repasse, se prend, se donne, se suit, se dédouble, en somme elle circule dans la chaine parlée. Le journaliste a pour rôle de canaliser le flux discursif du géographe, d'en endiguer les débordements, d'en guider le courant discursif.

Développer les compétences discursives de compréhension orale de l'apprenant demande donc de se concentrer sur cette dynamique, d'observer les tours de paroles, leur progression, leur progressivité afin de repérer et d'identifier les différentes postures énonciatives à l'œuvre dans le débat. Ce faisant, l'apprenant sera mieux à même de comprendre les subtilités énonciatives et discursives de l'interaction qui tissent la trame de la chaîne parlée dans ce type d'émission. C'est bien le cadre de la glottodidactique qui permettra d'intégrer la réflexion de l'analyse discursive à la didactique de la compréhension orale et d'initier un modèle de stratégie d'écoute opérant en contexte. 


\section{BIBLIOGRAPHIE}

Alvarez-Prendez, E. (2004). Le paradoxe linguistique : le cas des énoncés concessifs. In J. Suso, R. Lopez Carrillo (réd.), Le français face aux défis actuels. Histoire, langue et culture, vol. I (pp. 524-525). Granada : APFUE-GILEC.

Berrendonner, A. (2002). Portrait de l'énonciateur en faux naiff. Semen [En ligne], 15. URL: http://semen.revues.org/240

Blanche-Benveniste, C. (1990). Un modèle d'analyse syntaxique 'en grilles' pour les productions orales. Anuario de Psicologia, 47, 11-28.

Blanche-Benveniste, C., Bilger, M., Rouget, Ch., van den Eynde, K. (1990). Le français parlé : Études grammaticales. Paris : Éditions du CNRS.

Cadre Européen Commun de Référence pour les Langues : Conseil de l'Europe / Les Éditions Didier, Paris 2001. URL : http://www.coe.int/t/dg4/linguistic/Source/Framework_fr.pdf

Drouet, G. (sous presse). La contradiction en discours, analyse d'une mise en scène singulière. Paris : Classiques Garnier, collection Domaines linguistiques.

Górecka, J., Wilczyńska, W., Wojciechowska, B. (2015). Developing second language oral competence through an integrated discursive approach: The conceptual framework of the project and the pilot study results. In M. Pawlak, E. Waniek-Klimczak (réd.), Theoretical, empirical and pedagogic perspectives on teaching, learning and assessing speaking skills in a second language (pp. 29-43). Heidelberg, Berlin : Springer-Verlag.

Kerbrat-Orecchioni, C. (2005). Discours en interaction. Paris : Armand Colin.

Kerbrat-Orecchioni, C. (2012). Négocier la notion de négociation, Négociations, 1, 17, 87-99.

Maingueneau, D. (2014). Discours et analyse du discours. Paris : Armand Colin.

Richard, E., Wojciechowska, B., Le Bot, M.-C., Wilczyńska, W., Górecka, J., Bourvon, M.-F. (2014). Les débats radiophoniques : quelles stratégies d'écoute pour le niveau avancé ? In C. Martinot, A. Pegaz Paquet (réd.), Innovations didactiques en français langue étrangère (pp. 38-50). Paris : CRL éditions.

Vion, R. (1992). La Communication verbale. Analyse des interactions. Paris : Hachette Supérieur.

Wojciechowska, B. (2015). Perception et interprétation des émotions dans le débat radiodiffusé par les étudiants avancés de philologie romane. Studia Romanica Posnaniensia, XLII/4, 155-168.

Wojciechowska, B., Górecka, J., Richard, E., Le Bot, M.-C. (2014). Les stratégies rédactionnelles face aux défis d'interprétation d'un débat radiodiffusé, Studia Romanica Posnaniensia, XLI/3, 117-132.

Wojciechowska, B., Richard, E. (2016). Négociation interactive de l'implicite dans le débat polémique radiophonique », Corela HS-20 [en ligne]. URL: http://corela.revues.org/4684. Doi : 10.4000/ corela.4684 